\title{
ДОСТОВЕРНОСТЬ РЕЗУЛЬТАТОВ FRАХ У ЖЕНЩИН С ПОВТОРНЫМИ ПЕРЕЛОМАМИ ПО ДАННЫМ КЛИНИЧЕСКОЙ ПРАКТИКИ
}

\author{
Долгова К.С., Демидова Т.Ю.
}

Российский национальный исследовательский медицинский университет им. Н.И. Пирогова, Москва

Остеопороз является клинико-экономической проблемой. В России среди лиц в возрасте 50 лет и старше остеопороз выявляется у $34 \%$ женщин и $27 \%$ мужчин, а частота остеопении составляет $43 \%$ и $44 \%$ соответственно. Частота остеопороза увеличивается с возрастом. Для определения необходимости в антирезорбтивной терапии у пациентов с остеопорозом используют инструмент оценки риска перелома FRAX. По данным рекомендаций Российской ассоциации эндокринологов по остеопорозу, при наличии низкотравматических переломов (НП) рекомендовано устанавливать диагноз остеопороза и назначать лечение независимо от результатов денситометрии или FRAX. Мы проанализировали наличие факторов риска (ФР) развития остеопороза у пациентов в возрасте от 48 до 88 лет с имеющимися НП в анамнезе, оценили 10-летнюю вероятность развития НП и порог терапевтического вмешательства (та индивидуальная вероятность перелома, при котором оправдано начинать лечение) по FRAX, оценили риск падений у данных пациентов, выявили группу пациентов, которым необходимо получение антирезорбтивной терапии.

ЦЕЛЬ: Оценить достоверность результатов 10-летней вероятности развития НП и порога терапевтического вмешательтва у пациентов с имеющимися НП в анамнезе.

МАТЕРИАЛЫ И МЕТОДЫ: Проведен анализ данных 26 пациентов ГКБ им. В.П. Демихова в возрасте от 48 до 88 лет с имеющимися НП в анамнезе, которые ранее не получали терапию антирезорбтивными препаратами или прекратившие прием препаратов 2 и более года назад. Статистическая обработка полученных данных проведена при помощи Microsoft Excel.

РЕзУЛЬТАТЫ: В ходе исследования проведен анализ данных 26 женщин в постменопаузе с НП в возрасте от 48 до 88 лет. В исследовании были проанализированы ФР развития остеопороза: наследственность (перелом бедра у родителей) - у 26,923\%, курение - 19,231\%, сахарный диабет 2 типа - 26,923\%, ревматоидный артрит - 26,923\%, низкая физическая активность - 57,692\%, длительная иммобилизация - 30,769\%, ранняя менопауза и постменопаузальный период - 30,769\%, низкая масса тела в период набора пика костной массы - 15,385\%, избыточная масса тела - 69,231\%. Перелом шейки бедра был выявлен у 30,769\%, лодыжки - 23,077\%, плечевой кости - 19,231\%, лучевой кости - 15,385\% по данным рентгенографии. Снижение роста на 4 см за всю жизнь было выявлено у 23,077\%, что косвенно указывает на наличие компрессионного перелома позвоночника. 2 и более НП в анамнезе наблюдалось у 23,077\%. Однако, точка терапевтического вмешательства по данным FRAX была выявлена у 50\% пациентов, необходимость проведения денситометрии для подтверждения необходимости лечения - у 23,077\%, отсутствие необходимости назначения лечения и проведения денситометрии - у 23,077\%, что говорит о неполноценности данного FRAX. Возможно, это связано с тем, что такие ФР развития остеопороза как: 2 и более НП в анамнезе, наличие сахарного диабета 2 типа, низкая физическая активность, длительная иммобилизация, низкая масса тела в период набора пика костной массы не учитываются в данном опроснике.

Выводы: По результатам исследования было выявлено, что FRAX не дает достоверных результатов о необходимости лечения пациентов с низкоэнергетическими переломами. Необходима дальнейшая доработка FRAX с целью более точного выявления когорты пациентов с остеопорозом, требующих проведения антирезорбтивной терапии. 